Research Paper

\title{
Optimization of zofimarin production by an endophytic fungus, Xylaria sp. Acra L38
}

\author{
Jirapan Chaichanan ${ }^{1}$, Suthep Wiyakrutta ${ }^{1}$, Thunyarat Pongtharangkul ${ }^{2}$, \\ Duangnate Isarangkul $^{1}$, Vithaya Meevootisom ${ }^{1 *}$ \\ ${ }^{1}$ Department of Microbiology, Faculty of Science, Mahidol University, Rachathewi, Bangkok, Thailand. \\ ${ }^{2}$ Department of Biotechnology, Faculty of Science, Mahidol University, Rachathewi, Bangkok, Thailand.
}

Submitted: July 4, 2012; Approved: September 9, 2013.

\begin{abstract}
To optimize the medium for high zofimarin production, sucrose maltose, glucose, tryptone and peptone were used in an orthogonal array design experiment, where the highest value of zofimarin produced was $25.6 \mu \mathrm{g} / \mathrm{mL}$. This value was about 3 times higher than that obtained with Czapek yeast extract (CzYE) culture medium. A study with Plackett-Burman design showed that sucrose, maltose, glucose and $\mathrm{NaNO}_{3}$ were significant factors in zofimarin production. Further studies using central composite design (CCD) showed the significance of glucose and the interactions of these critical components affecting zofimarin production. Multiple regression analysis of the data yielded a poor fit as shown by the mismatch of the model with these variable factors. When a polynomial equation was applied, the maximum zofimarin production was predicted to be $201.9 \mu \mathrm{g} / \mathrm{mL}$. Experimental verification yielded a much lower amount of zofimarin, at around $70 \mu \mathrm{g} / \mathrm{mL}$. Reconsideration of the CCD data and repetition of some runs with high zofimarin production resulted in reproducible zofimarin yield at $79.7 \mu \mathrm{g} / \mathrm{mL}$. Even though the amount was lower than the predicted value, the medium optimization study was considered to be quite successful as the yield increased to around 8 times that obtained with the original CzYE culture medium.
\end{abstract}

Key words: zofimarin, secondary metabolite, medium optimization, statistical methods, endophytic fungus.

\section{Introduction}

Zofimarin was first discovered in 1987 from a marine fungus Zopfielle marina SANK21274 and was classified by Ogita et al. (1987) as a sordarin derivative due to their related chemical structures. It has an acyl group attached to the pyran ring of the tetracyclic aglycon common to all sordarins (Figure 1) (Hanadate et al., 2009).

The compound had strong antifungal activity against pathogenic yeasts, including azole-resistant Candida albicans. Its antifungal activity is based on the inhibition of fungal protein synthesis via binding to fungal eEF2, leading to an inhibition of the translocation step and finally led to cell death (Dominguez and Martin, 1998; Justice et al., 1997). Zofimarin and other sordarin derivatives exhibited high selectivity for eEF2 of fungi as compared with those of plants or mammals, despite the fact that their amino acid se- quences have high level of homology. This high selectivity and the mechanism of action make zofimarin one of the promising candidates as a lead compound in therapeutic drugs for the treatment of fungal infection (Sigg and Stoll, 1969). Since the risk of opportunistic fungal infections is increasing among immune-compromised people along with the emergence of drug resistant fungal pathogens, the search for potentially effective antifungal agents and lead compounds should be continued.

Recently, our research group successfully isolated an endophytic fungus, Xylaria sp. Acra L38, from a Thai medicinal plant, Aquilaria crassna (Wetwitaklung et al., 2009). The fungus produced an active compound having activity against $C$. albicans ATCC 90028 . Structural identification of the active compound by UV, IR and ${ }^{1} \mathrm{H}$ - and ${ }^{13} \mathrm{C}-\mathrm{NMR}$ spectroscopic analyses revealed it to be structurally identical to zofimarin. The yield of zofimarin produced 


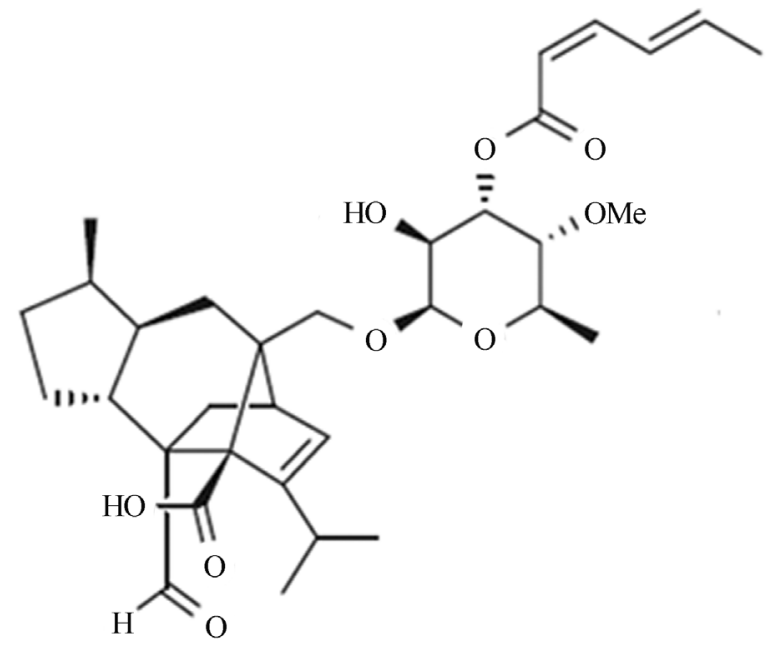

Figure 1 - Chemical structure of zofimarin.

by Acra L38 in Czapek yeast extract (CzYE) culture medium was relatively low $(<10 \mu \mathrm{g} / \mathrm{mL})$. Since zofimarin seemed to be a good candidate for a lead compound with high potential of use as a future therapeutic antifungal agent, a study was aimed to increase the fermentation yield using statistical approaches for optimization of the fermentation medium.

Suitable fermentation media were known to be important for increased production of secondary metabolites (Cai et al., 2009). Many successful media were optimized by employing statistical methods that systematically analyzed the important medium components and their interactions, instead of the conventional approach, which employed a full factorial design and single factor optimization (Box et al., 1978). Our previous study showed that Xylaria sp. Acra L38 could produce zofimarin only when $\mathrm{CzYE}$ was used as the fermentation medium. Thus, in this study, components in CzYE were first subjected to investigation using an orthogonal array design (OD) experiment to determine the optimum concentration of the medium components followed by Plackett-Burman design (PBD) to determine the key medium components. Finally, the response surface methodology (RSM) was adopted to investigate the effect of the significant medium components and their interactions affecting zofimarin production (Chang et al., 2006; Escamilla et al., 2000; Li et al., 2008). With this approach, the fermentation medium would be optimized to give a higher zofimarin yield.

\section{Materials and Methods}

\section{Chemicals}

Reagents purchased from Merck Chemical Company (Darmstadt, Germany) were glucose, peptone, yeast extract and various inorganic salts including $\mathrm{NaNO}_{3}$. Acetonitrile of HPLC purity was obtained from Fisher Scientific (Leicestershire, UK). Maltose was purchased from Difco
Laboratories (Michigan, USA) while sucrose was obtained from a local supermarket and tryptone was purchased from Dickinson and Company (New York, USA).

\section{Media}

Media were prepared by dissolving the ingredients in distilled water and sterilized by autoclaving at $121{ }^{\circ} \mathrm{C}$ for 15 min. Potato dextrose agar (PDA) was purchased from Merck Chemical Company. CzYE contained per liter $5 \mathrm{~g}$ yeast extract, $30 \mathrm{~g}$ sucrose, $0.3 \mathrm{~g} \mathrm{NaNO}_{3}, 0.05 \mathrm{~g} \mathrm{KCl}, 0.05 \mathrm{~g}$ $\mathrm{MgSO}_{4} .7 \mathrm{H}_{2} \mathrm{O}, 1 \mathrm{mg} \mathrm{FeSO}{ }_{4} .7 \mathrm{H}_{2} \mathrm{O}, 1 \mathrm{mg} \mathrm{ZnSO}_{4} .7 \mathrm{H}_{2} \mathrm{O}$, $0.5 \mathrm{mg} \mathrm{CuSO}{ }_{4} .5 \mathrm{H}_{2} \mathrm{O}$ and $1 \mathrm{~g} \mathrm{~K}_{2} \mathrm{HPO}_{4}$.

\section{Strain and inoculum preparation}

Xylaria sp. Acra L38 has been isolated by our laboratory in the Department of Microbiology, Faculty of Science, Mahidol University. The culture was maintained for daily work on PDA slants while the stock culture was kept at $-80{ }^{\circ} \mathrm{C}$. To prepare inoculums, Acra L38 was grown on PDA plates at $25^{\circ} \mathrm{C}$ for 12 days. Then agar plugs were cut from the edge of the colony by using a sterilized cork borer (no. 4) and used as inoculums.

\section{Fermentation and sampling}

Fermentation was done using $50 \mathrm{~mL}$ autoclave-sterilized fermentation broth in $250 \mathrm{~mL}$ Erlenmeyer flasks (Pyrex $^{\circledR} 4980$ ), which were plugged with cotton wool and covered with aluminum foil before sterilization. Four pieces of inoculum plugs were placed into each culture flask and incubated at $25^{\circ} \mathrm{C}$ under static conditions. One milliliter of the fermentation broth was aseptically withdrawn for HPLC analysis at specified times. All experiments were performed in duplicate unless specified otherwise.

\section{Analytical methods}

To determine zofimarin concentration, the withdrawn sample was diluted 2-fold with distilled water and centrifuged at $15,000 \mathrm{~g}$ for $2 \mathrm{~min}$. The supernatant was subjected to HPLC analysis using an Agilent LC 1100 HPLC system with the following conditions.

- Column: Hewlett-Packard ODS Hypersil, $5 \mu \mathrm{m}$, $125 \times 4 \mathrm{~mm}$ with LiChrosphere $100 \mathrm{RP}-18,5 \mathrm{~mm}$, $4 \times 4 \mathrm{~mm}$ guard column.

- Column temperature: Ambient $\left(25^{\circ} \mathrm{C}\right)$.

- Mobile phase: $0.1 \% \mathrm{AcOH}$ in $\mathrm{H}_{2} \mathrm{O}$ : acetonitrile (35:65).

- Flow rate: $2.0 \mathrm{~mL} / \mathrm{min}$.

- Injection volume: $20 \mu \mathrm{L}$.

- Detection: UV-photodiode array detector monitored at $268 \mathrm{~nm}$.

The run time for HPLC analysis was 8 min, where zofimarin was eluted at around $3.4 \mathrm{~min}$. Zofimarin concentration in the broth was calculated using the standard curve prepared with purified zofimarin, which was kindly pro- 
vided by Dr. Khanit Suwanborirux and Ms. Malinee Oyuchua.

\section{Experimental design and data analysis}

\section{Orthogonal array design}

Since previous study showed that Xylaria sp. Acra L38 could produce zofimarin only when CzYE was used as the fermentation medium, components in CzYE were subjected to OD study. Suitable concentrations of carbon sources (sucrose, maltose and glucose) and nitrogen sources (yeast extract, tryptone and peptone) were determined for high zofimarin production using an OD designed experiment, $\mathrm{L}_{25}\left(5^{6}\right)$, which was based on five levels of carbon sources $(5,10,15,20$ and $25 \mathrm{~g} / \mathrm{L})$ and nitrogen sources $(4,6,8,10$ and $12 \mathrm{~g} / \mathrm{L})$. Other components were kept at the same level as in CzYE.

\section{Plackett-Burman design}

In order to determine key medium components for zofimarin production, PBD was employed to analyze 7 independent components (variables) in 12 combinations. The experimental runs were designed using Design Expert (version 7.0, Stat-Ease, Inc., Minnesota, USA). Analysis of variance (ANOVA) was used for data analysis. Each experimental variable was tested for a high value $(+1)$ and low value $(-1)$. The low value was obtained from the previous OD experiment and the high value was 1.25 times higher. Therefore, the high levels of the medium compositions used in PBD were $(\mathrm{g} / \mathrm{L})$ : sucrose 12 , maltose 6.25 , glucose 12.5 , yeast extracts 10 , tryptone 12.5 , peptone 15 and $\mathrm{NaNO}_{3} 0.125$.

\section{Response surface methodology}

To maximize zofimarin production, four significant factors (sucrose, maltose, glucose and $\mathrm{NaNO}_{3}$ ) identified by the PBD experiment were investigated using a CCD experiment covering thirty runs with six replicates at the center point. The concentrations of each component at the center point were confirmed using the single gradient test, in which concentration of the response component was varied while those of the other components were kept constant. The concentrations used in the single gradient test were taken from the combination giving the highest zofimarin yield in the PBD study. Then, the optimum concentrations of all significant components were used as the center point for CCD with five levels $(-2,-1,0,1,2)$. The yield of zofimarin was taken as the response $(Y)$. The regression equation model was determined for robustness under the determination coefficient $\left(R^{2}\right)$ and adjusted determination coefficient ( $\operatorname{adj} R^{2}$ ). Experimental design and data analysis were performed using Minitab software release 15 (Minitab Inc., State College, PA, USA).

\section{Results and Discussion}

\section{Orthogonal array design}

OD was employed first to obtain preliminary suitable concentrations of carbon and nitrogen sources as well as to reduce the scope of experimental runs. Twenty-five runs with 5 concentration levels were performed as shown in Table 1 . The results showed that some of the combinations did not produce zofimarin at all while combinations 6 and 11 resulted in high zofimarin production. Thus, subsequent experiments were designed to optimize these medium components. Based on combination 6 with highest zofimarin production $(25.6 \mu \mathrm{g} / \mathrm{mL})$, low values $(-1)$ of all components for PBD were established as follows $(\mathrm{g} / \mathrm{L})$ : sucrose 10 , maltose 5 , glucose 10 , yeast extracts 8 , tryptone 10 and peptone 12 .

\section{Plackett-Burman design}

To determine the critical medium components and their effects on zofimarin production, three carbon sources (sucrose, maltose and glucose), three nitrogen sources (yeast extract, tryptone and peptone) and $\mathrm{NaNO}_{3}$ were investigated using Plackett-Burman design. It should be noted that $\mathrm{NaNO}_{3}$ was included in this study because it has been reported as a good nitrogen source for zofimarin production in our previous study (unpublished data). From an analysis of variance (ANOVA), p-values indicated the significance of each factor (Table 2). At 95\% confidence level, the corresponding factor was considered to be significant when $p$-value $<0.05$ (10). The critical medium components identified included sucrose, maltose, glucose and $\mathrm{NaNO}_{3}$ (Table 2). Economically, the use of $\mathrm{NaNO}_{3}$ as nitrogen source instead of costly yeast extract and peptone would reduce the medium cost dramatically. In the next experiment, interactions between these critical factors (sucrose, maltose, glucose and $\mathrm{NaNO}_{3}$ ) will be elucidated by the response surface method.

\section{Response surface method}

CCD was used to investigate the interactions among the significant medium components identified by the Plackett-Burman experiment. Appropriate center point values of all significant factors (sucrose, maltose, glucose and $\mathrm{NaNO}_{3}$ ) were determined using a single factor gradient test. Results from the gradient study suggested the center points for sucrose, maltose, glucose and $\mathrm{NaNO}_{3}$ at 7.5, 5, 15 and $0.01 \mathrm{~g} / \mathrm{L}$, respectively (Figure 2 ).

In this CCD study, five concentration levels $(-2,-1,0$, $1,2)$ were established based on the center point values previously determined (Table 3 ). The results from CCD were then applied to multiple regression analysis using a second-order polynomial equation. Based on the p-values obtained (Table 4), glucose was found to be the most significant factor affecting zofimarin production. This was expected because glucose is the most readily utilized carbon 
Table 1 - Orthogonal array for sucrose, maltose, glucose, yeast extract, tryptone and peptone (g/L).

\begin{tabular}{|c|c|c|c|c|c|c|c|}
\hline No. & Sucrose & Maltose & Glucose & Yeast extract & Tryptone & Peptone & Zofimarin $(\mu \mathrm{g} / \mathrm{mL})$ \\
\hline 1 & 5 & 5 & 5 & 4 & 4 & 4 & 9.6 \\
\hline 2 & 5 & 10 & 10 & 6 & 6 & 6 & 7.0 \\
\hline 3 & 5 & 15 & 15 & 8 & 8 & 8 & 5.8 \\
\hline 4 & 5 & 20 & 20 & 10 & 10 & 10 & 0.0 \\
\hline 5 & 5 & 25 & 25 & 12 & 12 & 12 & 0.0 \\
\hline 6 & 10 & 5 & 10 & 8 & 10 & 12 & 25.6 \\
\hline 7 & 10 & 10 & 15 & 10 & 12 & 4 & 4.80 \\
\hline 8 & 10 & 15 & 20 & 12 & 4 & 6 & 3.8 \\
\hline 9 & 10 & 20 & 25 & 4 & 6 & 8 & 0.0 \\
\hline 10 & 10 & 25 & 5 & 6 & 8 & 10 & 0.0 \\
\hline 11 & 15 & 5 & 15 & 12 & 6 & 10 & 23.0 \\
\hline 12 & 15 & 10 & 20 & 4 & 8 & 12 & 0.0 \\
\hline 13 & 15 & 15 & 25 & 6 & 10 & 4 & 0.0 \\
\hline 14 & 15 & 20 & 5 & 8 & 12 & 6 & 0.0 \\
\hline 15 & 15 & 25 & 10 & 10 & 4 & 8 & 2.7 \\
\hline 16 & 20 & 5 & 20 & 6 & 12 & 8 & 8.1 \\
\hline 17 & 20 & 10 & 25 & 8 & 4 & 10 & 0.0 \\
\hline 18 & 20 & 15 & 5 & 10 & 6 & 12 & 8.9 \\
\hline 19 & 20 & 20 & 10 & 12 & 8 & 4 & 0.0 \\
\hline 20 & 20 & 25 & 15 & 4 & 10 & 6 & 2.1 \\
\hline 21 & 25 & 5 & 25 & 10 & 8 & 6 & 0.0 \\
\hline 22 & 25 & 10 & 5 & 12 & 10 & 8 & 11.4 \\
\hline 23 & 25 & 15 & 10 & 4 & 12 & 10 & 10.4 \\
\hline 24 & 25 & 20 & 15 & 6 & 4 & 12 & 0.0 \\
\hline 25 & 25 & 25 & 20 & 8 & 6 & 4 & 0.0 \\
\hline Range & 26.5 & 2.5 & 42.1 & 23.8 & 37.6 & 28.1 & \\
\hline
\end{tabular}
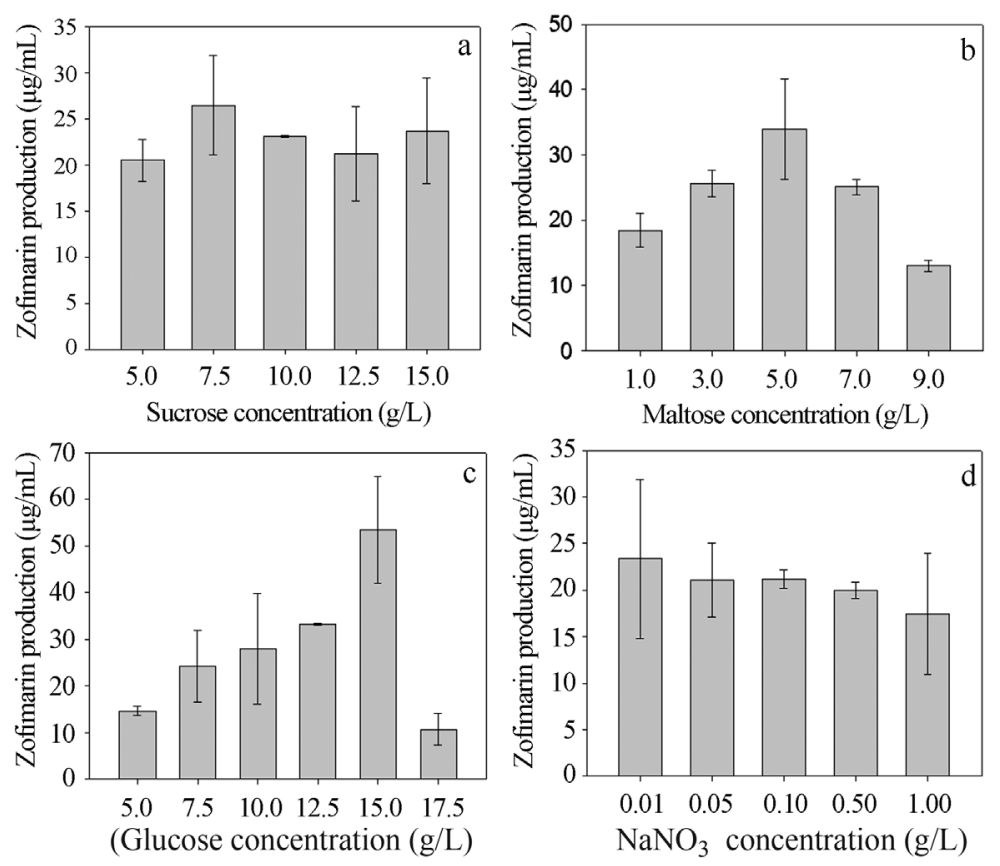

Figure 2 - Effects of single gradient tests on zofimarin production, for sucrose (a), maltose (b), glucose (c) and $\mathrm{NaNO}_{3}(\mathrm{~d})$. 
Table 2 - ANOVA test of Plackett-Burman result.

\begin{tabular}{|c|c|c|c|c|}
\hline Source & $\begin{array}{l}\text { Mean } \\
\text { square }\end{array}$ & F-value & p-value & Effect \\
\hline Corrected model & 138.493 & 9.394 & 0.023 & \\
\hline Intercept & 6932.223 & 470.216 & 0.000 & \\
\hline Sucrose & 241.876 & 16.407 & 0.015 & -8.68 \\
\hline Maltose & 277.835 & 18.846 & 0.012 & -9.62 \\
\hline Glucose & 229.644 & 15.577 & 0.017 & 8.75 \\
\hline Yeast & 22.296 & 1.512 & 0.286 & 2.75 \\
\hline Tryptone & 35.024 & 2.376 & 0.198 & 3.42 \\
\hline Peptone & 42.725 & 2.898 & 0.164 & 3.75 \\
\hline $\mathrm{NaNO}_{3}$ & 120.049 & 8.143 & 0.046 & -6.35 \\
\hline Error & 14.743 & 470.216 & 0.000 & -8.68 \\
\hline
\end{tabular}

R Squared $=0.943 ;$ Adjusted R Squared $=0.842$. source allowing good growth, but on the other hand it might repress zofimarin production due to glucose catabolite repression (Feng et al., 1994; Gupta et al., 2007).

The multiple regression analysis revealed $R^{2}$ of 0.709 , indicating that the model adequately represents the data. Nevertheless, the statistically significant lack of fit (LOF) from ANOVA analysis indicated either that the model was not fitting all the design points well or the responses were highly repetitive around the center point (where the pure error was derived from) or both. Similar observation was also reported by Oh et al. (1995). Despite the significant lack of fit, prediction of zofimarin yield was attempted. Maximum zofimarin production was predicted to be $201.9 \mu \mathrm{g} / \mathrm{mL}$ at $2.5 \mathrm{~g} / \mathrm{L}$ maltose, $25 \mathrm{~g} / \mathrm{L}$ glucose and $20 \mathrm{mg} / \mathrm{L} \mathrm{NaNO}_{3}$.

To verify reliability of the predicted result, Acra L38 was cultured using the predicted combination in triplicate.

Table 3 - Central composite design for sucrose, maltose, glucose and $\mathrm{NaNO}_{3}$ on zofimarin production.

\begin{tabular}{|c|c|c|c|c|c|c|}
\hline \multirow[t]{2}{*}{ No. } & \multirow[t]{2}{*}{ Sucrose $(g / L)$} & \multirow[t]{2}{*}{ Maltose (g/L) } & \multirow[t]{2}{*}{ Glucose (g/L) } & \multirow[t]{2}{*}{$\mathrm{NaNO}_{3}(\mathrm{mg} / \mathrm{L})$} & \multicolumn{2}{|c|}{ Zofimarin $(\mu \mathrm{g} / \mathrm{mL})$} \\
\hline & & & & & A & $\mathrm{B}$ \\
\hline 1 & 5 & 2.5 & 10 & 15 & 23.0 & 33.2 \\
\hline 2 & 5 & 2.5 & 20 & 5 & 44.1 & 44.9 \\
\hline 3 & 5 & 7.5 & 10 & 5 & 50.2 & 51.3 \\
\hline 4 & 5 & 7.5 & 20 & 15 & 52.2 & 60.4 \\
\hline 5 & 10 & 2.5 & 10 & 5 & 27.2 & 20.8 \\
\hline 6 & 10 & 2.5 & 20 & 15 & 71.1 & 85.5 \\
\hline 7 & 10 & 7.5 & 10 & 15 & 47.9 & 54.6 \\
\hline 8 & 10 & 7.5 & 20 & 5 & 72.1 & 89.9 \\
\hline 9 & 7.5 & 5 & 15 & 10 & 41.8 & 46.4 \\
\hline 10 & 7.5 & 5 & 15 & 10 & 31.1 & 30.7 \\
\hline 11 & 5 & 2.5 & 10 & 5 & 23.8 & 44.8 \\
\hline 12 & 5 & 2.5 & 20 & 15 & 94.9 & 107.6 \\
\hline 13 & 5 & 7.5 & 10 & 15 & 34.0 & 37.0 \\
\hline 14 & 5 & 7.5 & 20 & 5 & 43.2 & 49.9 \\
\hline 15 & 10 & 2.5 & 10 & 15 & 23.4 & 25.2 \\
\hline 16 & 10 & 2.5 & 20 & 5 & 74.7 & 68.7 \\
\hline 17 & 10 & 7.5 & 10 & 5 & 47.2 & 45.8 \\
\hline 18 & 10 & 7.5 & 20 & 15 & 46.6 & 46.6 \\
\hline 19 & 7.5 & 5 & 15 & 10 & 68.3 & 60.3 \\
\hline 20 & 7.5 & 5 & 15 & 10 & 34.8 & 59.1 \\
\hline 21 & 2.5 & 5 & 15 & 10 & 74.3 & 57.8 \\
\hline 22 & 12.5 & 5 & 15 & 10 & 61.0 & 74.0 \\
\hline 23 & 7.5 & 0 & 15 & 10 & 41.0 & 54.6 \\
\hline 24 & 7.5 & 10 & 15 & 10 & 62.0 & 49.5 \\
\hline 25 & 7.5 & 5 & 5 & 10 & 14.8 & 22.0 \\
\hline 26 & 7.5 & 5 & 25 & 10 & 77.0 & 72.0 \\
\hline 27 & 7.5 & 5 & 15 & 0 & 79.0 & 60.2 \\
\hline 28 & 7.5 & 5 & 15 & 20 & 32.3 & 44.2 \\
\hline 29 & 7.5 & 5 & 15 & 10 & 40.1 & 41.1 \\
\hline 30 & 7.5 & 5 & 15 & 10 & 41.1 & 40.1 \\
\hline
\end{tabular}

The full quadratic model giving the highest adjusted $R^{2}(0.61)$ was selected:

$\mathrm{Y}=44.7708+0.9508$ (sucrose $)+0.7375$ (maltose $)+14.5862$ (glucose $)-1.4175\left(\mathrm{NaNO}_{3}\right)-5.0438(\text { sucrose })^{2}+1.2981(\text { maltose })^{2}-0.0356(\text { glucose })^{2}+$ $1.8325\left(\mathrm{NaNO}_{3}\right)^{2}+3.2719$ (sucrose)(maltose) +1.585 (sucrose) $($ glucose $)-4.625($ sucrose $)\left(\mathrm{NaNO}_{3}\right)-9.0681$ (maltose) $($ glucose $)-6.1819$ (maltose $)\left(\mathrm{NaNO}_{3}\right)+3.8462$ (sucrose $)\left(\mathrm{NaNO}_{3}\right)$, where $Y$ is zofimarin concentration $(\mu \mathrm{g} / \mathrm{mL})$. 
Table 4 - Regression analysis for CCD design.

\begin{tabular}{|c|c|c|c|c|}
\hline Term & Coef & SE Coef & $\mathrm{T}$ & $\mathrm{p}$ \\
\hline Constant & 44.7708 & 3.721 & 12.033 & 0.000 \\
\hline Block & -0.6101 & 1.743 & -0.350 & 0.728 \\
\hline A & 0.9508 & 1.838 & 0.517 & 0.607 \\
\hline B & 0.7375 & 1.838 & 0.401 & 0.690 \\
\hline $\mathrm{C}$ & 14.5862 & 1.838 & 7.938 & 0.000 \\
\hline $\mathrm{D}$ & -1.4175 & 1.838 & -0.771 & 0.445 \\
\hline$A^{*} \mathrm{~A}$ & 5.0438 & 1.719 & 2.934 & 0.005 \\
\hline $\mathrm{B} * \mathrm{~B}$ & 1.2981 & 1.719 & 0.755 & 0.454 \\
\hline $\mathrm{C}^{*} \mathrm{C}$ & -0.0356 & 1.719 & -0.021 & 0.984 \\
\hline$D * D$ & 1.8325 & 1.719 & 1.066 & 0.292 \\
\hline$A * B$ & 3.2719 & 2.250 & 1.454 & 0.153 \\
\hline$A^{*} \mathrm{C}$ & 1.5850 & 2.250 & 0.704 & 0.485 \\
\hline$A * D$ & -4.6250 & 2.250 & -2.055 & 0.046 \\
\hline $\mathrm{B}^{*} \mathrm{C}$ & -9.0681 & 2.250 & -4.029 & 0.000 \\
\hline$B^{*} \mathrm{D}$ & -6.1819 & 2.250 & -2.747 & 0.009 \\
\hline $\mathrm{C}^{*} \mathrm{D}$ & 3.8462 & 2.250 & 1.709 & 0.094 \\
\hline $\mathrm{S}=12.7306$ & & PRESS $=13441.3$ & & \\
\hline $\mathrm{R}-\mathrm{Sq}=70.88 \%$ & & $\mathrm{R}-\mathrm{Sq}($ pred $)=45.12 \%$ & & $\mathrm{R}-\mathrm{Sq}(\operatorname{adj})=60.96 \%$ \\
\hline
\end{tabular}

The analysis was done using coded units.

Estimated regression coefficients for response.

Sucrose (A), maltose (B), glucose (C) and $\mathrm{NaNO}_{3}$ (D).

The average zofimarin yield obtained was only $60.8 \mu \mathrm{g} / \mathrm{mL}$, which was much lower than the predicted value. This observation is consistent with a low predicted $R^{2}$ observed from the model $(0.451)$, confirming that the model cannot predict new observations nearly as well as it fits the existing data. Therefore, the CCD data were reconsidered and experimental run numbers $6,8,12$ and 26 giving high level of zofimarin were repeated experimentally in triplicate. Of the four medium compositions, the highest zofimarin production $(79.7 \mu \mathrm{g} / \mathrm{mL})$ was observed from composition 26. Thus, zofimarin production using the medium composition newly developed in this study was 8 times higher than that obtained with the original $\mathrm{CzYE}$ cul- ture medium. The 8-fold increase is somewhat comparable to what were reported for sordarin, alkaline lipase, cytosine-substituted mildiomycin analogue, and lutein as 25 , 12, 3.8 and 5, respectively (Dan et al., 2008; Gupta et al., 2007; Shinde and Lele, 2010; Tully et al., 2007).

In conclusion, the medium composition was optimized using several statistical designs for zofimarin production by the endophytic fungus Xylaria sp. Acra L38. Starting with an OD experiment, the highest yield of zofimarin obtained was $25.6 \mu \mathrm{g} / \mathrm{mL}$, approximately 3 times higher than that obtained with the original $\mathrm{CzYE}$ culture medium. Followed with PBD, sucrose, maltose, glucose and $\mathrm{NaNO}_{3}$ were identified as significant factors for zofi-

Table 5 - Analysis of variance for response.

\begin{tabular}{|c|c|c|c|c|c|c|}
\hline Source & DF & Seg SS & Adj SS & Adj MS & $\mathrm{F}$ & $\mathrm{p}$ \\
\hline Blocks & 1 & 19.9 & 19.9 & 19.85 & 0.12 & 0.728 \\
\hline Regression & 14 & 17340.6 & 17340.6 & 1238.61 & 7.64 & 0.000 \\
\hline Linear & 4 & 10378.4 & 10378.4 & 2594.59 & 16.01 & 0.000 \\
\hline Square & 4 & 1527.0 & 1527.0 & 381.76 & 2.36 & 0.068 \\
\hline Interaction & 6 & 5435.1 & 5435.1 & 905.86 & 5.59 & 0.000 \\
\hline Residual Error & 44 & 7131.0 & 7131.0 & 162.07 & & \\
\hline Lack-of-Fit & 10 & 4325.3 & 4325.3 & 432.53 & 5.24 & 0.000 \\
\hline Pure Error & 34 & 2805.7 & 2805.7 & 82.52 & & \\
\hline Total & 59 & 24491.4 & & & & \\
\hline
\end{tabular}


marin production. Further study with CCD showed that glucose was the most significant factor affecting zofimarin production of Acra L38 and significant interactions were observed among sucrose, maltose, glucose and $\mathrm{NaNO}_{3}$. Although the quadratic equation failed to predict the maximum zofimarin production, reconsideration of the CCD data and repetition of selected combinations with high zofimarin concentration resulted in reproducible zofimarin production at $79.7 \mu \mathrm{g} / \mathrm{mL}$, more than 8 times higher than that obtained with the original $\mathrm{CzYE}$ culture medium.

\section{Acknowledgments}

This work was supported by Mahidol University research grant. We also thank Dr. Khanit Suwanborirux, Department of Pharmacognosy and Pharmaceutical Botany, Faculty of Pharmaceutical Sciences, Chulalongkorn University and Ms. Malinee Oyuchua, Department of Microbiology, Faculty of Science, Mahidol University for providing purified zofimarin.

\section{References}

Box G, Hunter J, Hunter W (1978) Statistics for experimenters: An introduction to design, data analysis, and model building. John Wiley \& Sons Inc., New York.

Cai MH, Zhou XS, Sun XQ, Tao KJ, Zhang YX (2009) Statistical optimization of medium composition for aspergiolide A production by marine-derived fungus Aspergillus glaucus. J Ind Microbiol Biotechnol 36:381-389.

Chang MY, Tsai GJ, Houng JY (2006) Optimization of the medium composition for the submerged culture of Ganoderma lucidum by Taguchi array design and steepest ascent method. Enzyme Microb Technol 38:407-414.

Dan Y, Zhi-nan X, Pei C (2008) Medium optimization for enhanced production of cytosine-substituted mildiomycin analogue (MIL-C) by Streptoverticillium rimofaciens ZJU 5119. J Zhejiang Univ Sci B 9:77-84.

Dominguez JM, Martin JJ (1998) Identification of elongation factor 2 as the essential protein targeted by sordarin in Candida albicans. Antimicrob. Agents Chemother 42:2279-2283.

Escamilla EM, Dendooven L, Magana IP, Parra R, De la Torre M (2000) Optimization of gibberellic acid production by immobilized Gibberella fujikuroi mycelium in fluidized bioreactors. J Biotechnol 76:147-155.
Feng B, Friedlin E, Marzlufi GA (1994) A reporter gene analysis of penicillin biosynthesis gene expression in Penicillium chrysogenum and its regulation by nitrogen and glucose catabolite repression. Appl Environ Microbiol 60:44324439.

Hanadate T, Tomishima M, Shiraishi N, Tanabe D, Morikawa H, Barrett D, Matumoto S, Ohtomo K, Maki K (2009) FR290581, a novel sordarin derivative: Synthesis and antifungal activity. Bioorg Med Chem Lett 19:1465-1468.

Justice M, Hsu MJ, Tse B, Ku T, Balkovec J, Schmatz D, Nielsen J (1997) Elongation factor 2 as a novel target for selective inhibition of fungal protein synthesis. J Biol Chem 273:31483151.

Li XY, Liu ZQ, Chi ZM (2008) Production of phytase by a marine yeast Kodamaea ohmeri BG3 in an oats medium: Optimization by response surface methodology. Bioresource Technol 99:6386-6390.

Gupta NV, Sahai V, Gupta R (2007) Alkaline lipase from a novel strain Burkholderia multivorans: Statistical medium optimization and production in a bioreactor. Process Biochem 42:518-526.

Ogita T, Hayashi A, Sato S, Furaya K, February (1987) Antibiotic zofimarin. Jpn Kokai Tokkyo Koho JP 6240292.

Oh S, Rheem S, Sim J, Kim S, Baek Y (1995) Optimizing conditions for the growth of Lactobacillus casei YIT 9018 in tryptone-yeast extract-glucose medium by using response surface methodology. Appl Environ Microbiol 61:38093814 .

Shinde SD, Lele SS (2010) Statistical media optimization for lutein production from microalgae Auxenochlorella protothecoides SAG 211-7A. Int J Adv Biotechnol Res 1:104-114.

Sigg HP, Stoll C March (1969) Antibiotic SL, 2266. US patent US 3432598 .

Tully TP, Bergum JS, Schwarz SR, Durand SC, Howell JM, Patel RN, Cino PM (2007) Improvement of sordarin production through process optimization: combining traditional approaches with DOE. J Ind Microbiol Biotechnol 34:193202.

Wetwitaklung P, Thavanapong N, Charoenteeraboon J (2009) Chemical constituents and antimicrobial activity of essential oil and extracts of heartwood of Aquilaria crassna obtained from water distillation and supercritical fluid carbon dioxide extraction. Silpakorn Univ Sci Tech J 3:25-33.

All the content of the journal, except where otherwise noted, is licensed under a Creative Commons License CC BY-NC. 